\title{
Rational Strategies for Combining Bcl-2 Inhibition with Targeted Drugs for Anti-Tumor Synergy
}

\author{
Erin Schwab', Justin A. Chen', Jasmine C. Huynh', Jingran Ji', Mili Arora', May Cho', Edward J. Kim* \\ 'Department of Internal Medicine, University of California Davis, USA
}

Article Info

Article Notes

Received: August 28, 2019

Accepted: October 10, 2019

\section{*Correspondence:}

Dr. Edward J. Kim, UC Davis Comprehensive Cancer Center, 4501 X Street, Suite 3016, Sacramento, CA 95817 USA;

Telephone No: (916) 734-3772; Fax No: (916) 734-7946; Email: jhkim@ucdavis.edu.

C $2019 \mathrm{Kim}$ EJ. This article is distributed under the terms of the Creative Commons Attribution 4.0 International License.

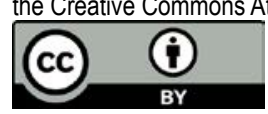

\section{Keywords}

Bcl-2

$\mathrm{BH} 3$

Apoptosis

Synergy

Anti-Tumor

Tumorigenesis

\section{ABSTRACT}

Based on the well-established importance of dysregulated apoptosis as a hallmark of cancer, there has been robust interest in development of targeted drugs to promote cancer cell apoptosis. A promising target for promoting apoptosis is the $\mathrm{Bcl}-2$ family of proteins. Bcl-2 family proteins are crucial in maintaining balance between cell survival and death through regulation of apoptotic signaling pathways via pro-survival and pro-apoptotic family members. To date, there has been limited efficacy with Bcl-2 inhibition alone in clinical development with benefit restricted to hematologic malignancies. However, combination approaches to inhibition of $\mathrm{Bcl}-2$ and other oncogenic signaling pathways have provided evidence for potential anti-tumor synergy. We review herein the current evidence for targeting $\mathrm{Bcl}-2$ family proteins as a cancer therapeutic strategy across both hematologic and solid organ malignancies.

\section{Introduction}

B-cell lymphoma 2 (Bcl-2) family proteins are an integral player in intrinsic cellular apoptosis. The extrinsic apoptotic pathway is mediated by cell surface receptors, while intrinsic apoptosis involves mitochondria. The Bcl-2 family balance of cell survival and death regulates mitochondrial apoptotic signaling pathways which influence mitochondrial permeability. This family of proteins contain $\mathrm{Bcl}-2$ homology $(\mathrm{BH})$ domains that are imperative for heterodimeric interactions between $\mathrm{Bcl}-2$ family members ${ }^{1}$.

Bcl-2 family members are categorized into three groups: prosurvival Bcl-2 proteins, death effector proteins, and pro-apoptotic BH3-only proteins. Pro-survival, anti-apoptotic members include $\mathrm{Bcl}-2$, Bcl-xL, Bcl-w, Bcl-b, Mcl-1, and Bcl-2A1 and share four regions of sequence homology (BH1-4) $)^{2,3}$. Overexpression of any of the prosurvival members leads to cellular protection against apoptosis, even in the presence of cytotoxic stimuli ${ }^{4}$. Death effector proteins which lack the BH4 domain include Bax, Bak, and Bok. These are sequestered by pro-survival Bcl-2 proteins under normal conditions. Pro-apoptotic BH3-only proteins include BIM, BIK, PUMA, BAD, NOXA, and BID; they remain inactive until a cytotoxic stimulus ${ }^{3}$. When a stimulus occurs, $\mathrm{BH} 3$-only proteins insert the $\mathrm{BH} 3$ domains into the BH3-binding grooves of the pro-survival Bcl-2 proteins causing release of Bax and Bak. These death effector proteins induce pore formation in the mitochondrial outer membrane which releases second mitochondria-derived activator of caspase (Smac, Diablo) and cytochrome c. This event activates the caspase cascade to ultimately trigger apoptosis ${ }^{5,6}$.One mechanism of apoptotic escape and tumorigenesis is through the overexpression of pro-survival Bcl-2 family members ${ }^{5,6}$. 


\section{Role of Bcl-2 in tumorigenesis}

Overexpression of $\mathrm{Bcl}-2$ has been classically demonstrated in B-cell and follicular lymphomas via a translocation between chromosome 14 and 18 leading to a fused immunoglobulin heavy chain and $\mathrm{Bcl}-2 \mathrm{loci}^{7-9}$. Bcl-2 expression has been suggested to have prognostic value in solid organ malignancies. In prostate cancer, Bcl-2 expression has been correlated to castration resistance and disease progression ${ }^{10}$. In breast cancer, Bcl-2 positivity on immunohistochemistry may indicate lower 10 -year overall survival ${ }^{11}$. Expression of $\mathrm{Bcl}-2$ is regulated by methylation and NF- $\kappa \mathrm{B}$ of the promotor region, with hypomethylation having been demonstrated in lymphoproliferative neoplasms ${ }^{12,13}$.

Pre-clinical models of have suggested that overexpression of Bcl-2 may be more permissive than causative in tumorigenesis. Transgenic mice with $t(14 ; 18)$ were initially observed to express a lower grade indolent lymphoma; however, after a prolonged latency period, disease often progressed to aggressive variants associated with additional genetic abnormalities such as c-myc rearrangement ${ }^{14}$. Similar findings of prolonged latency but eventual development of aggressive B-cell lymphomas have been seen with Mcl-1 transgenic mice ${ }^{15}$. Bcl-2 family proteins are additionally implicated in actively promoting tumor survival, as Mcl-1 is known to be critically important in survival of multiple myeloma cell lines ${ }^{16}$. Though $t(14 ; 18)$ is not seen outside of hematologic malignancies, Bcl-2 overexpression is seen in other cancers including solid organ malignancies.

Similarly to overexpression of Bcl-2, suppression of pro-apoptotic genes leads to a permissive rather than causative effects on tumorigenesis ${ }^{17}$. TP53 is a well-known tumor suppressor gene that is also implicated in regulation of apoptosis. Cytotoxic stimuli typically upregulate TP53 to induce apoptosis; however, this regulatory pathway is often lost in malignancies with mutated TP5 $3^{18}$. PUMA, or p53 upregulated mediator of apoptosis, is a BH3-only proapoptotic protein that is a downstream effector of p53mediated apoptosis. PUMA expression has been implicated in the progression of malignant melanoma, with lower expression corresponding to more advanced disease and worse prognosis ${ }^{19}$.

\section{Targeting Bcl-2 for cancer therapy}

Strategies for targeting Bcl-2-associated pathways in cancer include antisense oligonucleotides which neutralizes gene expression at the mRNA level and BH3 mimetics to promote apoptosis. The latter category typically includes small molecular inhibitors against anti-apoptotic proteins ${ }^{3}$.

Antisense oligonucleotides such as oblimersen have been evaluated in chronic lymphocytic leukemia (CLL) up through phase III clinical trials either as monotherapy or in combination with chemotherapy. Modest responses were seen with monotherapy and improved upon with various combinations of fludarabine, cyclophosphamide, and rituximab. Unfortunately, toxicity even in monotherapy limits its use with the most common adverse events being infusion reactions with or without tumor lysis, fever, fatigue, cytopenias, cough, and hypotension ${ }^{20}$.

Peptide sequences mimicking BH3-only proteins have been developed and found to bind to $\mathrm{Bcl}-2$ with higher affinity than pro-apoptotic proteins, affecting their interaction. Those pro-apoptotic proteins then homogenize, triggering apoptotic cascades with mitochondrial pore formation. One of these peptides was derived from the BH3 domain on BAX, inhibiting the BAX-Bcl-2 formation ${ }^{21}$. Unfortunately, these proteins were limited in scope due to degradation by cellular proteases and poor cell permeability and metabolic instability. Several studies have tried to overcome the instability of the protein by methods such as adding hydrocarbons to form a stable alpha-helix of BLC2 domains. This addition was shown to inhibit growth of leukemia xenografts, but these molecules have not progressed to clinical trials ${ }^{22}$.

The third and most successful strategy in drug development has been the development of Bcl-2 small molecule inhibitors. As with the peptides, these inhibitors have a high affinity to pro-survival proteins like Bcl2, Bcl-xl, and Bcl-w ${ }^{23,24}$. Thus, BH3 mimicking small molecule inhibitors work by blocking the Bcl-2 and BAK/ BAX interaction. Homology modeling predicted Bcl-2 protein structure by employing the Bcl-XL in complex with Bak BH3 peptide as a template. This method led to the development of HA14-1, the first small molecule Bcl-2 inhibitor developed by computer-based modeling ${ }^{25}$. Since then, many other Bcl-2 inhibitors, including gossypol and ABT-199, have been developed.

\section{BH3 mimetics as mono-therapy}

As mentioned above, small molecular inhibitors have had to the most success with anti-cancer effects in clinical trials. Gossypol is a naturally occurring polyphenolic compound found in cotton plants and had shown to inhibit apoptosis in mouse models ${ }^{26}$. It acts as a pan-Bcl-2 inhibitor and comes in two enantiomers; the negative enantiomer, referred to as AT-101, was found to be more cytotoxic via Bcl-2 inhibition, reactive oxygen species (ROS) generation and DNA cleavage ${ }^{27}$. It was shown to be efficacious in a wide variety of tumor types including breast, prostate, and non-small cell lung cancer (NSCLC). Unfortunately, this benefit did not extend to phase II clinical trials. Low binding affinity and toxic side effects limited this option.

Obatoclax is another pan-Bcl-2 inhibitor that has not demonstrated efficacy in phase I clinical trials for hematologic malignancies and is associated with neurologic 
toxicities $^{28}$. ABT-737 is a high-affinity inhibitor of Bcl-2, $\mathrm{Bcl}-\mathrm{xL}$, and Bcl-w that mechanistically mimics Bad BH3 peptides to inhibit anti-apoptotic Bcl-2 family proteins. Pre-clinical efficacy has been demonstrated in cell lines and patient-derived xenograft PDX murine models of follicular lymphoma, CLL, and small cell lung cancer. Further development was limited by poor oral availability ${ }^{29}$. Its successor navitoclax (ABT263) is an orally administered inhibitor of Bcl-2, Bcl-xL, and Bcl-w. Navitoclax had promising results as a single agent against relapsed/ refractory CLL in a phase I trial with a partial response rate of 35\% and median progression free survival of 25 months. The main dose-limiting toxicity was thrombocytopenia, which has been attributed to inhibition of $\mathrm{Bcl}-\mathrm{xL}^{30}$.

Venetoclax (ABT-199) is a selective Bcl-2 inhibitor without activity against Bcl-xL and Bcl-w. By sparing Bcl$\mathrm{xL}$, venetoclax is thought to cause less thrombocytopenia compared to navitoclax ${ }^{31}$. A phase I clinical trial of venetoclax monotherapy proved efficacy in relapsed/ refractory CLL with a $79 \%$ response rate, $20 \%$ complete remission rate, and $5 \%$ minimal residual disease-negative rate along with a 15-month progression-free survival (PFS) of $69 \%$ in patients receiving $400 \mathrm{mg}$ daily ${ }^{32}$. This led to FDA approval of venetoclax for CLL as single agent for relapsed CLL in April 2016.

\section{Combinatorial approaches to $\mathrm{BH} 3$ mimetic therapy}

Exploiting apoptosis through Bcl-2 family proteins with other modalities of systemic therapy is an active area of research across a wide variety of malignancies. Other oncogenic signaling pathways such as MEK-ERK, PI3KAKT, BRAF, KRAS, and EGFR have been associated with the Bcl-2-mediated apoptotic cascade, thus combinatorial approaches are an attractive strategy to augment responses to Bcl-2 family-directed therapies ${ }^{33-35}$.

\section{Combination with Endocrine Therapy in Estrogen Receptor (ER)-positive Breast Cancer}

The protein product Bcl-2 is overexpressed in $85 \%$ of cases of ER-positive early breast cancers and nearly $75 \%$ of cases in all early breast cancers ${ }^{34,36}$. Pre-clinical studies examining Bcl-2 inhibition with endocrine therapy have shown promise ${ }^{37}$. ER-positive xenograft mouse models were treated with either ABT-737 alone, tamoxifen alone, or combined ABT-737 and tamoxifen. ABT-737 alone had minimal effect but combined therapy demonstrated slowed tumor progression and complete response in a subset of xenografts. The mechanism of synergy was attributed to increased apoptosis - responsive xenografts had higher levels of cleaved caspase- 3 and combinatorial therapy mitigated the Bcl-2 and BIM upregulation seen with tamoxifen therapy. Despite higher levels of Bcl-xl also seen with tamoxifen therapy, the selective Bcl-2 inhibitor ABT-199 with tamoxifen showed similar cell viability and tumor growth delay compared to ABT-737 with tamoxifen in ER-positive cell lines and xenografts. Thrombocytopenia was seen more in ABT-737 than ABT-199 therapy owing to Bcl-xL sparing in the latter. Resistant xenograft models to combined tamoxifen and Bcl-2 inhibition demonstrated higher pAKT levels suggesting a role for the PI3K/AKT/ mTOR pathway. Similar to tamoxifen, mTOR inhibition upregulated Bcl-2 expression. Cell lines treated with ABT737 or ABT-199 and a PI3K/mTOR inhibitor AZD8055 resulted in synergistic, caspase-dependent cell death. Triple therapy of Bcl-2 inhibition, PI3K/mTOR inhibition, and tamoxifen in xenografts demonstrated synergistic reduction of tumor growth and prolonged survival. Interestingly, ABT-737 reduced endometrial thickness whereas ABT-199 did not, suggesting a role of Bcl-xl in tamoxifen-induced endometrial hyperplasia. This is an important finding given known pro-estrogenic side effects of tamoxifen on the endometrium with tamoxifen monotherapy.

A phase Ib dose escalation study of 33 patients has examined the role of Bcl-2 inhibitor ABT-199 (venetoclax) combined with tamoxifen in ER-positive, Bcl-2-positive metastatic breast cancer ${ }^{38}$. Venetoclax and tamoxifen therapy resulted in a clinical benefit rate (partial response + complete response + stable disease) of $70 \%$, time to objective response of 12 weeks, and median duration of response 42 weeks. The expansion phase recruited 24 patients and resulted in a clinical benefit rate of $75 \%$ with an objective response rate (ORR) of $54 \%$. Correlatives included measurement of circulating tumor DNA (ctDNA) at baseline and during therapy, with serial ctDNA measurements corresponding to clinical response. At baseline, PIK3CA (42\%) and ESR1 (30\%) were the most commonly detected abnormalities. Although no particular mutations seemed predictive of this treatment strategy, response was present in patients with an ESR1 mutation - a mutation which is an important mechanism for endocrine resistance. The most common adverse events included grade 1-2 nausea (67\%) and cytopenias, predominantly lymphopenia (88\%; grade $\geq 330 \%$ ).

\section{Combination with MEK inhibitors in KRAS-mutated NSCLC}

KRAS is a frequent driver mutation in multiple solid organ malignancies including non-small cell lung cancer. KRAS exerts oncogenic effects via the MAPK pathway (also known as the RAS/RAF/MEK/ERK pathway) which ultimately converges with the PI3K/AKT pathway on Bcl2-mediated apoptosis to suppress the pro-apoptotic BH3 protein BIM. MEK inhibitors increase BIM expression but its apoptotic activity can be inhibited by Mcl-1 and Bcl$\mathrm{xl}$. Therefore, combined MEK and Mcl-1 inhibition is a promising strategy that has been examined in in vitro and in vivo experiments ${ }^{39}$. 
Combinations of the MEK inhibitor trametinib with either the Mcl-1 inhibitor AM8621, navitoclax, or Bcl-xl inhibitor A1331852 have been shown to decrease cell viability. Venetoclax, however, did not demonstrate this level of synergy suspected due to its Bcl-2 selectivity. Therefore, exploiting pro-apoptotic pathways with MEK inhibition likely depends on Mcl-1 and Bcl-xl, and not Bcl2. Sensitivity to MEK and Mcl-1 or Bcl-xl inhibition in cell lines was not clearly dependent on baseline levels of Mcl-1 or Bcl-xl, respectively. Patient-derived xenografts of KRASmutated NSCLC models treated with trametinib and Mcl-1 inhibitors showed greater tumor response than trametinib alone or trametinib with navitoclax. Interestingly, when KRAS-mutated cell lines were treated first with a Bcl$\mathrm{xl}$ inhibitor, this increased BIM-Mcl-1 binding while decreasing levels of Bcl-xl. Sequential treatment using a Bcl-xl inhibitor and then adding Mcl-1 and MEK inhibition yielded reduced cell viability and enhanced tumor regression of xenografts when compared to non-pretreated specimens. By targeting Bcl-xl first, tumor survival become increasingly dependent on $\mathrm{Mcl}-1$ and therefore are primed against $\mathrm{Mcl}-1$-directed therapy.

\section{Combination with hypomethylating agents in acute myeloid leukemia (AML)}

Venetoclax monotherapy has been trialed in AML to modest efficacy ${ }^{40}$. Hypomethylating agents (HMAs) such as decitabine and azacitidine are thought to additionally inhibit Mcl-1 thus providing synergism between venetoclax and $\mathrm{HMAs}^{41}$.

HMAs plus venetoclax have been examined in the first-line setting for AML in elderly or frail patients unfit for standard induction ${ }^{42}$. Complete remission (CR) and complete remission with incomplete hematologic recovery (CRi) rates were $37 \%$ and $30 \%$, respectively, with ORR 68\% and leukemia response rate $(\mathrm{CR}+\mathrm{CRi}+$ partial response + morphologic leukemic free state) $83 \%$. Of patients with $\mathrm{CR}$ and $\mathrm{CRi}$, median duration of response was 11.3 months (95\% CI 8.9 m-NR). Overall median overall survival (OS) was 17.5 months (95\% CI 12.3 m-NR). Responses were seen across multiple cytogenetic and mutation subgroups although patients with adverse risk cytogenetics and TP53 had lower rates of response. Adverse events were mostly attributed to hematologic abnormalities, many of which were present at enrollment.

Prior to the development of this treatment strategy, single agent HMAs were standard-of-care in elderly or unfit newly-diagnosed AML patients. Previously reported efficacy outcomes have included median OS of 10 months, CR + CRi rates of $25-30 \%$, and median duration of CR + CRi 10 months $^{43}$. The Bcl-2 selective inhibitor venetoclax in combination with hypomethylating agents has therefore been a practice-changing therapeutic strategy which received accelerated FDA approved for first-line treatment of AML in elderly patients or those unfit for standard induction.

\section{Combination with XPO1 in Glioblastoma}

Chromosome region maintenance 1 (CRM1) also known as exportin 1 (XPO1) is a transporter of tumor suppressor proteins and its role in oncogenesis have been described in both hematologic and solid organ malignancies ${ }^{44}$. Increased expression of XPO1 has been postulated to move tumor suppressor proteins from the nucleus to cytoplasm where they cannot regulate the cell cycle ${ }^{45}$. Selinexor or KPT-330 is an oral selective inhibitor of XP01 that crosses the blood-brain barrier and therefore has been tested as monotherapy for recurrent glioblastomas ${ }^{46}$.

$\mathrm{BAG} 3$ or BIS is a $\mathrm{Bcl}-2$ interacting protein that synergizes with $\mathrm{Bcl}-2$ to prevent apoptosis. Its expression typically portends a worse prognosis in glioblastomas ${ }^{47-49}$. Preclinical glioblastoma models have demonstrated improved apoptotic response to BH3 mimetics with concurrent BAG3 silencing or depletion ${ }^{47}$. Selinexor with a variety of BH3 mimetics including navitoclax, venetoclax, WEHI-539 (Bcl-xl inhibitor), and A1210477 (Mcl-1 inhibitor) has been shown to be effective in glioblastoma cell lines and patient-derived xenografts. Combination therapy reduces cellular proliferation and increases sensitivity to selinexor via enhanced apoptosis as demonstrated through DNA fragmentation, annexin staining, mitochondrial membrane disruption, and cleaved caspases ${ }^{49}$. Navitoclax alone was seen to increase Mcl-1 levels; by adding selinexor, Mcl-1 protein levels were then suppressed. Similar effects were noted in PDX models of glioblastoma with combined therapy of selinexor and navitoclax demonstrating greater growth inhibition than either therapy alone likely due to broad inhibition of pro-apoptotic proteins.

\section{Combination with BRAF inhibitor}

$\mathrm{BRAF}^{\mathrm{V} 600 \mathrm{E}}$-mutated malignancies generally respond to the $\mathrm{BRAF}^{\mathrm{V} 600 \mathrm{E}}$-specific inhibitors, and the combination of dabrafenib and trametinib has moved into the first-line setting for advanced $\mathrm{BRAF}^{\mathrm{V} 600 \mathrm{E}}$-mutated melanoma ${ }^{50-52}$. In melanoma, median progression-free survival to singleagent vemurafenib tends to be brief at around 7 months. This is improved with the addition of MEK inhibition to 11 months ${ }^{52}$. While the addition of MEK inhibition prolongs response duration, resistant pathways inevitably develop thus limiting the efficacy of BRAF-targeted treatment ${ }^{53}$.

Navitoclax has been evaluated with vemurafenib in $\mathrm{BRAF}^{\mathrm{V} 600 \mathrm{E}}$-mutated thyroid cancer cell lines ${ }^{54}$. Navitoclax alone had no effect on cellular survival. Vemurafenib alone reduced cell viability by $10.4 \%$ and also increased expression of Bcl-2 and Bcl-xl 24 hours after exposure. In contrast, the combination reduced cell viability by 
$39.4 \%$ and increased apoptotic activity. Since navitoclax is both a Bcl-2 and Bcl-xl inhibitor, its addition to BRAF inhibitor therapy represents a potential method of mitigating resistance that may develop under single agent vemurafenib.

Similar treatment strategies of combined BRAF inhibition with BH3 mimetics have been evaluated in $\mathrm{BRAF}^{\mathrm{V} 600 \mathrm{E}}$-mutated melanoma ${ }^{54,55}$. In patients treated with either vemurafenib or dabrafenib and trametinib, pretreatment tumor mRNA levels of Bcl-2 were inversely correlated to response to BRAF-directed therapy. Initiating therapy did not change Bcl-2 levels but did increase mRNA levels of BIM, Bcl-xl, Bcl-w, BID mRNA levels while decreasing mRNA levels of $\mathrm{Mcl}-1^{55}$. The changes in their respective protein expression levels were not necessarily consistent with changes seen at the mRNA level.

In pre-clinical models of cell lines and xenografts, navitoclax plus PLX4720 (BRAF inhibitor) resulted in increased cell death and BIM-mediated apoptotic activity compared to either therapy alone ${ }^{55,56}$. Patient-derived melanoma cell lines pre-BRAF treatment seemed sensitive to BH3 mimetic plus BRAF inhibition; however, melanoma cells that have already developed BRAF inhibitor resistance were additionally resistant to combined therapy with a BH3 mimetic ${ }^{56}$. Additionally, resistant cell lines have been observed to express higher levels of anti-apoptotic Mcl$1^{56}$. This suggests combined therapy may have limited efficacy against tumors pretreated with BRAF inhibition, and resistance against combined therapy may be driven by Mcl-1.

\section{Combination with Aurora Kinase Inhibitor}

The aurora kinases are a family of serine/threonine kinases that include Aurora Kinase A (AKA), B, and C. While all three have the potential for tumorigenesis, AKA has been the most well-studied, and its overexpression is associated with genomic instability, abnormal G2/M checkpoint transition, mitotic disruption, and aberrant activation of other oncogenic signaling pathways ${ }^{57}$.

Alisertib or MLN 8237 is an AKA inhibitor that has had little success as monotherapy and is therefore being increasingly examined in combination therapy. Alisertib has been observed to reduce Mcl-1 levels, and since Mcl1 is a potential resistance mechanism to single agent BH3 mimetic therapy ${ }^{58}$, the addition of alisertib to $\mathrm{BH} 3$ mimetics is an attractive combinatorial therapy strategy. Pancreatic cancer cell lines, organoids, and xenografts exposed to either alisertib or navitoclax alone had variable sensitivity to each; however, the combination yielded increased growth inhibition. Apoptotic activity with this approach was confirmed with higher levels of cleaved caspase-3, cleaved caspase-7, cleaved PARP, and annexin V. Furthermore, pancreatic cancer organoids undergoing treatment with MLN 8237 alone demonstrated reduced Mcl-1 levels while treatment with navitoclax alone demonstrated increased Mcl-1 levels and to a lesser extent Bcl-xl levels. The addition of MLN 8237 to navitoclax counteracted the Mcl-1 upregulation seen with navitoclax only ${ }^{58}$. Therefore, the synergistic effect of this combined therapy may be attributable to AKA inhibition effects on Mcl-1.

\section{Combination therapy in CLL}

Ibrutinib is a Bruton's tyrosine kinase inhibitor approved for front-line therapy of CLL. Mechanistically, ibrutinib is thought to mobilize CLL cells thus removing them from a pro-survival microenvironment. Additional effects on the apoptotic pathway includes reduced levels of $\mathrm{Mcl}-1$ and $\mathrm{Bcl}-\mathrm{xl}^{59}$. Thus the combination of ibrutinib and venetoclax is a potential strategy in CLL that has now been tested in a phase II clinical trial for first-line therapy of $\mathrm{CLL}^{60}$. Of 80 evaluable patients, $74 \%$ of patients were able to achieve CR or CRi. Of the 33 patients that received 12 cycles of combination therapy, 29 patients (88\%) were in CR or CRi and 20 patients (69\%) had no minimal residual disease in the bone marrow. One-year PFS and OS were $98 \%$ and 99\%, respectively. Responses were seen across high-risk subgroups. Adverse events included atrial fibrillation, neutropenia, and neutropenic fever at similar rates to prior published data with ibrutinib monotherapy ${ }^{32}$. Laboratory tumor lysis was seen in only 3 patients. While the combination is a promising front-line option, longer follow-up and randomized trials examining this strategy compared to either in monotherapy are warranted.

As discussed previously, single agent venetoclax is a potential option for patients with relapsed/refractory CLL. Resistance to this strategy may be attributable to elevated levels of pro-survival Bcl-2 family proteins such as survivin, Mcl-1, Bcl-xl, and Bfl-1 ${ }^{61,62}$. Similar favoring of pro-survival proteins has been seen with CD40 stimulation in vitro ${ }^{62,63}$; however, exposure of anti-CD20 treatment to CD40-stimulated CLL cells causes lysosome-mediated cell death $^{64,65}$. This concept has translated into clinical trials examining venetoclax with CD20 antibody therapy ${ }^{66,67}$. The combination of venetoclax plus rituximab has been examined in the relapsed/refractory setting compared to bendamustine plus rituximab demonstrating 2-year median PFS of $84.9 \%$ compared to $36.3 \%$, respectively (HR 0.17 , 95\% CI 0.11-0.25, p<0.001) ${ }^{66}$. Moreover, follow-up analyses have shown deeper and more sustained remissions with venetoclax plus rituximab. Peripheral blood undetectable minimal residual disease rates were $62 \%$ compared to $13 \%$ and 3-year median PFS rates were $71 . \%$ compared to $15.2 \%{ }^{68}$. This benefit was seen regardless of poor-risk features such as del(17p), TP53, unmutated IGHV. Rates of grade 3 or 4 neutropenia and tumor lysis were slightly higher in the venetoclax plus rituximab arm. 
For patients with baseline renal dysfunction or other medical co-morbidities as determined by the Cumulative Illness Rating Score, venetoclax plus obinutuzumab has been compared to chlorambucil plus obinutuzumab in the frontline setting in a phase III clinical trial ${ }^{67}$. The venetoclax/ obinutuzumab arm had a 2-year median PFS of $88.2 \%$ compared to $64.1 \%$ for chlorambucil/obinutuzumab, and again the benefit was seen across poor-riskfeatures. Toxicity profile between the two arms were similar. Therefore, the addition of anti-CD20 therapy to venetoclax is synergistic in its clinical effect and mechanistically mitigates potential resistance to venetoclax alone by promoting both apoptotic and lysosomal cell death.

\section{Conclusion}

The Bcl-2 family of proteins is an important regulator of intrinsic apoptosis and thus represents an attractive target for cancer-directed therapy. BH3 mimetics and small molecular inhibitors of pro-apoptotic proteins is an increasingly exploited therapeutic strategy that has moved into the first-line setting for hematologic malignancies including AML and CLL. Combinatorial approaches with other agents such as chemotherapy, inhibitors of other oncologic driver mutations, or monoclonal antibodies offers synergistic anti-tumor effects that likely offset resistant mechanisms seen with $\mathrm{BH} 3$ mimetic therapy alone. These agents exhibit significant potential to improve current therapies in hematologic and non-hematologic malignancies.

\section{References}

1. Chittenden T, Harrington EA, O'Connor R, et al. Induction of apoptosis by the Bcl-2 homologue Bak. Nature. 1995; 374: 733-6.

2. Czabotar PE, Lessene G, Strasser A, et al. Control of apoptosis by the BCL-2 protein family: implications for physiology and therapy. Nat Rev Mol Cell Biol. 2014; 15: 49-63.

3. Radha G, Raghavan SC. BCL2: A promising cancer therapeutic target. Biochim Biophys Acta Rev Cancer. 2017; 1868: 309-14.

4. Cory S, Huang DC, Adams JM. The Bcl-2 family: roles in cell survival and oncogenesis. Oncogene. 2003; 22: 8590-607.

5. Anderson MA, Huang D, Roberts A. Targeting BCL2 for the treatment of lymphoid malignancies. Semin Hematol. 2014; 51: 219-27.

6. Juin P, Geneste $\mathrm{O}$, Gautier F, et al. Decoding and unlocking the BCL-2 dependency of cancer cells. Nat Rev Cancer. 2013; 13: 455-65.

7. Tsujimoto Y, Cossman J, Jaffe E, et al. Involvement of the bcl-2 gene in human follicular lymphoma. Science. 1985; 228: 1440-3.

8. Tsujimoto Y, Finger LR, Yunis J, et al. Cloning of the chromosome breakpoint of neoplastic B cells with the $t(14 ; 18)$ chromosome translocation. Science. 1984; 226: 1097-9.

9. Cleary ML, Sklar J. Nucleotide sequence of a t(14;18) chromosomal breakpoint in follicular lymphoma and demonstration of a breakpointcluster region near a transcriptionally active locus on chromosome 18. Proc Natl Acad Sci U S A. 1985; 82: 7439-43.

10. Catz SD, Johnson JL. BCL-2 in prostate cancer: a minireview. Apoptosis. 2003; 8: 29-37.

11. Callagy GM, Pharoah PD, Pinder SE, et al. Bcl-2 is a prognostic marker in breast cancer independently of the Nottingham Prognostic Index. Clin Cancer Res. 2006; 12: 2468-75.

12. Hanada M, Delia D, Aiello A, et al. bcl-2 gene hypomethylation and high-level expression in B-cell chronic lymphocytic leukemia. Blood. 1993; 82: 1820-8.

13. Catz SD, Johnson JL. Transcriptional regulation of bcl-2 by nuclear factor kappa B and its significance in prostate cancer. Oncogene. 2001; 20: 7342-51.

14. McDonnell TJ, Korsmeyer SJ. Progression from lymphoid hyperplasia to high-grade malignant lymphoma in mice transgenic for the $t(14$; 18). Nature. 1991; 349: 254-6.

15. Zhou P, Levy NB, Xie HY, et al. MCL1 transgenic mice exhibit a high incidence of B-cell lymphoma manifested as a spectrum of histologic subtypes. Blood. 2001; 97: 3902-9.

16. Zhang B, Gojo I, Fenton RG. Myeloid cell factor-1 is a critical survival factor for multiple myeloma. Blood. 2002; 99: 1885-93.

17. Elkholi R, Floros KV, Chipuk JE. The Role of BH3-Only Proteins in Tumor Cell Development, Signaling, and Treatment. Genes Cancer. 2011; 2: 523-37.

18. Donehower LA, Lozano G. 20 years studying p53 functions in genetically engineered mice. Nat Rev Cancer. 2009; 9: 831-41.

19. Karst AM, Dai DL, Martinka M, et al. PUMA expression is significantly reduced in human cutaneous melanomas. Oncogene. 2005; 24: 1111-6.

20. Cheson BD. Oblimersen for the treatment of patients with chronic lymphocytic leukemia. Ther Clin Risk Manag. 2007; 3: 855-70.

21. Shangary S, Johnson DE. Peptides derived from BH3 domains of Bcl2 family members: a comparative analysis of inhibition of $\mathrm{Bcl}-2, \mathrm{Bcl}-$ $\mathrm{x}(\mathrm{L})$ and Bax oligomerization, induction of cytochrome c release, and activation of cell death. Biochemistry. 2002; 41: 9485-95.

22. Walensky LD, Pitter K, Morash J, et al. A stapled BID BH3 helix directly binds and activates BAX. Mol Cell. 2006; 24: 199-210.

23. Lessene G, Czabotar PE, Colman PM. BCL-2 family antagonists for cancer therapy. Nat Rev Drug Discov. 2008; 7: 989-1000.

24. Vela L, Marzo I. Bcl-2 family of proteins as drug targets for cancer chemotherapy: the long way of BH3 mimetics from bench to bedside. Curr Opin Pharmacol. 2015; 23: 74-81.

25. Wang JL, Liu D, Zhang ZJ, et al. Structure-based discovery of an organic compound that binds Bcl-2 protein and induces apoptosis of tumor cells. Proc Natl Acad Sci U S A. 2000; 97: 7124-9.

26. Wolter KG, Wang SJ, Henson BS, et al. (-)-gossypol inhibits growth and promotes apoptosis of human head and neck squamous cell carcinoma in vivo. Neoplasia. 2006; 8: 163-72.

27. Bai L, Wang S. Targeting apoptosis pathways for new cancer therapeutics. Annu Rev Med. 2014; 65: 139-55.

28. Liu Q Wang HG. Anti-cancer drug discovery and development: Bcl-2 family small molecule inhibitors. Commun Integr Biol. 2012; 5: 557-65.

29. Oltersdorf T, Elmore SW, Shoemaker AR, et al. An inhibitor of Bcl-2 family proteins induces regression of solid tumours. Nature. 2005; 435: 677-81.

30. Roberts AW, Seymour JF, Brown JR, et al. Substantial susceptibility of chronic lymphocytic leukemia to BCL2 inhibition: results of a phase I study of navitoclax in patients with relapsed or refractory disease. J Clin Oncol. 2012; 30: 488-96.

31. Souers AJ, Leverson JD, Boghaert ER, et al. ABT-199, a potent and selective BCL-2 inhibitor, achieves antitumor activity while sparing platelets. Nat Med. 2013; 19: 202-8.

32. Roberts AW, Davids MS, Pagel JM, et al. Targeting BCL2 with Venetoclax in Relapsed Chronic Lymphocytic Leukemia. New Engl J Med. 2016; 374: 311-22. 
33. Cragg MS, Jansen ES, Cook M, et al. Treatment of B-RAF mutant human tumor cells with a MEK inhibitor requires Bim and is enhanced by a BH3 mimetic. J Clin Invest. 2008; 118: 3651-9.

34. Deng J, Shimamura T, Perera S, et al. Proapoptotic BH3-only BCL-2 family protein BIM connects death signaling from epidermal growth factor receptor inhibition to the mitochondrion. Cancer Res. 2007; 67: 11867-75.

35. Hata AN, Yeo A, Faber AC, et al. Failure to Induce Apoptosis via BCL-2 Family Proteins Underlies Lack of Efficacy of Combined MEK and PI3K Inhibitors for KRAS-Mutant Lung Cancers. Cancer Research. 2014; 74: 3146-56.

36. Dawson SJ, Makretsov N, Blows FM, et al. BCL2 in breast cancer: a favourable prognostic marker across molecular subtypes and independent of adjuvant therapy received. Br J Cancer. 2010; 103: 668-75.

37. Vaillant F, Merino D, Lee L, et al. Targeting BCL-2 with the BH3 mimetic ABT-199 in estrogen receptor-positive breast cancer. Cancer Cell. 2013; 24: 120-9.

38. Lok SW, Whittle JR, Vaillant F, et al. A Phase Ib Dose-Escalation and Expansion Study of the BCL2 Inhibitor Venetoclax Combined with Tamoxifen in ER and BCL2-Positive Metastatic Breast Cancer. Cancer Discov. 2019; 9: 354-69.

39. Nangia V, Siddiqui FM, Caenepeel S, et al. Exploiting MCL1 Dependency with Combination MEK + MCL1 Inhibitors Leads to Induction of Apoptosis and Tumor Regression in KRAS-Mutant Non-Small Cell Lung Cancer. Cancer Discov. 2018; 8: 1598-613.

40. Konopleva M, Pollyea DA, Potluri J, et al. Efficacy and Biological Correlates of Response in a Phase II Study of Venetoclax Monotherapy in Patients with Acute Myelogenous Leukemia. Cancer Discov. 2016; 6: 1106-17.

41. Tsao T, Shi Y, Kornblau S, et al. Concomitant inhibition of DNA methyltransferase and BCL-2 protein function synergistically induce mitochondrial apoptosis in acute myelogenous leukemia cells. Ann Hematol. 2012; 91: 1861-70.

42. DiNardo CD, Pratz K, Pullarkat V, et al. Venetoclax combined with decitabine or azacitidine in treatment-naive, elderly patients with acute myeloid leukemia. Blood. 2019; 133: 7-17.

43. Dombret H, Seymour JF, Butrym A, et al. International phase 3 study of azacitidine vs conventional care regimens in older patients with newly diagnosed AML with $>30 \%$ blasts. Blood. 2015; 126: 291-9.

44. Wang AY, Liu $H$. The past, present, and future of CRM1/XPO1 inhibitors. Stem Cell Investig. 2019; 6: 6.

45. Hing ZA, Fung HYJ, Ranganathan P, et al. Next-generation XPO1 inhibitor shows improved efficacy and in vivo tolerability in hematological malignancies. Leukemia. 2016; 30: 2364-72.

46. Lassman AB, Bent MJVD, Wen PY, et al. Interim Analysis Data from Phase 2 Study on Efficacy, Safety \& Intratumoral Pharmacokinetics of Oral Selinexor (Kpt-330) in Patients with Recurrent Glioblastoma (Gbm). Neuro-Oncology. 2017; 19: 14-.

47. Antonietti P, Linder B, Hehlgans S, et al. Interference with the HSF1/ HSP70/BAG3 Pathway Primes Glioma Cells to Matrix Detachment and BH3 Mimetic-Induced Apoptosis. Mol Cancer Ther. 2017; 16: 156-68.

48. Lee JH, Takahashi T, Yasuhara N, et al. Bis, a Bcl-2-binding protein that synergizes with Bcl-2 in preventing cell death. Oncogene. 1999; 18: 6183-90.

49. Shang EY, Zhang YR, Shu C, et al. Dual Inhibition of Bcl-2/Bcl-xL and XP01 is synthetically lethal in glioblastoma model systems. Sci RepUk. 2018; 8.

50. Chapman PB, Hauschild A, Robert C, et al. Improved Survival with Vemurafenib in Melanoma with BRAF V600E Mutation. New Engl J Med. 2011; 364: 2507-16.
51. Kim KB, Cabanillas ME, Lazar AJ, et al. Clinical Responses to Vemurafenib in Patients with Metastatic Papillary Thyroid Cancer Harboring BRAF(V600E) Mutation. Thyroid. 2013; 23: 1277-83.

52. Robert C, Karaszewska B, Schachter J, et al. Improved Overall Survival in Melanoma with Combined Dabrafenib and Trametinib. New Engl J Med. 2015; 372: 30-9.

53. Kakadia S, Yarlagadda N, Awad R, et al. Mechanisms of resistance to BRAF and MEK inhibitors and clinical update of US Food and Drug Administration-approved targeted therapy in advanced melanoma. Onco Targets Ther. 2018; 11: 7095-107.

54. Jeong JH, Oh JM, Jeong SY, et al. Combination Treatment with the BRAF(V600E) Inhibitor Vemurafenib and the BH3 Mimetic Navitoclax for BRAF-Mutant Thyroid Carcinoma. Thyroid. 2019; 29: 540-8.

55. Frederick DT, Salas Fragomeni RA, Schalck A, et al. Clinical profiling of BCL-2 family members in the setting of BRAF inhibition offers a rationale for targeting de novo resistance using $\mathrm{BH} 3$ mimetics. PLoS One. 2014; 9: e101286.

56. Wroblewski D, Mijatov B, Mohana-Kumaran N, et al. The BH3-mimetic ABT-737 sensitizes human melanoma cells to apoptosis induced by selective BRAF inhibitors but does not reverse acquired resistance. Carcinogenesis. 2013; 34: 237-47.

57. Huynh J, Chen J, Arora M, et al. Strategic Combinations of Aurora Kinase an Inhibiton with Targeted Drugs for Synergistic Anti-Tumor Effect. Journal of Cancer Research and Cellular Therapeutics. 2019.

58. Duan Z, Chinn D, Tu MJ, et al. Novel Synergistic Combination of Mitotic Arrest and Promotion of Apoptosis for Treatment of Pancreatic Adenocarcinoma. Transl Oncol. 2019; 12: 683-92.

59. Cervantes-Gomez F, Lamothe B, Woyach JA, et al. Pharmacological and Protein Profiling Suggests Venetoclax (ABT-199) as Optimal Partner with Ibrutinib in Chronic Lymphocytic Leukemia. Clinical Cancer Research. 2015; 21: 3705-15.

60. Jain N, Keating M, Thompson P, et al. Ibrutinib and Venetoclax for First-Line Treatment of CLL. N Engl J Med. 2019; 380: 2095-103.

61. Smit LA, Hallaert DY, Spijker R, et al. Differential Noxa/Mcl-1 balance in peripheral versus lymph node chronic lymphocytic leukemia cells correlates with survival capacity. Blood. 2007; 109: 1660-8.

62. Tromp JM, Tonino SH, Elias JA, et al. Dichotomy in NF-kappaB signaling and chemoresistance in immunoglobulin variable heavy-chainmutated versus unmutated CLL cells upon CD40/TLR9 triggering. Oncogene. 2010; 29: 5071-82.

63. Thijssen R, Slinger E, Weller K, et al. Resistance to ABT-199 induced by microenvironmental signals in chronic lymphocytic leukemia can be counteracted by CD20 antibodies or kinase inhibitors. Haematologica. 2015; 100: e302-6.

64. Jak M, van Bochove GG, Reits EA, et al. CD40 stimulation sensitizes CLL cells to lysosomal cell death induction by type II anti-CD20 mAb GA101. Blood. 2011; 118: 5178-88.

65. Jak M, van Bochove GG, van Lier RA, et al. CD40 stimulation sensitizes CLL cells to rituximab-induced cell death. Leukemia. 2011; 25: 968-78.

66. Seymour JF, Kipps TJ, Eichhorst B, et al. Venetoclax-Rituximab in Relapsed or Refractory Chronic Lymphocytic Leukemia. N Engl J Med. 2018; 378: 1107-20.

67. Fischer K, Al-Sawaf O, Bahlo J, et al. Venetoclax and Obinutuzumab in Patients with CLL and Coexisting Conditions. N Engl J Med. 2019; 380: 2225-36.

68. Kater AP, Seymour JF, Hillmen P, et al. Fixed Duration of VenetoclaxRituximab in Relapsed/Refractory Chronic Lymphocytic Leukemia Eradicates Minimal Residual Disease and Prolongs Survival: PostTreatment Follow-Up of the MURANO Phase III Study. J Clin Oncol. 2019; 37: 269-77. 